\title{
Q\&A with Brenda Finucane: Building pipelines for genetic tests for autism
}

\section{BY ANGIE VOYLES ASKHAM}

17 AUGUST 2021

When Brenda Finucane began her work as a genetic counselor, she and her colleagues had few DNA testing tools at their disposal. They mainly relied on karyotyping, which involves looking at blurry images of a person's chromosomes under a microscope for any irregularities. But this technique could help them spot autism-related variants in only about 3 percent of autistic children who were tested, she says.

Recent advances in technology enable geneticists to detect more subtle variations in DNA. All told, they can find genetic variants linked to autism in about 25 percent of people with the condition information that has led to clinical diagnoses and personal benefits for those individuals and their families.

Since 2013, the American College of Medical Genetics and Genomics has recommended that all autistic boys and men and some autistic girls and women be tested for fragile $X$ syndrome. It also recommends that all autistic people undergo a chromosomal microarray test to identify any deleted or duplicated segments of their chromosomes. And in July, the organization updated those guidelines, suggesting that children with intellectual disability or other developmental disabilities also undergo whole-exome sequencing to spot more fine-grained genetic changes.

Only 3 percent of autistic children get the recommended tests, however. And many autistic people and their families aren't even aware that testing is an option.

"It's very frustrating," Finucane says. "People aren't going to ask for something they don't know is available."

Several obstacles prevent people from being tested, says Finucane, who is now associate director of the Autism and Developmental Medicine Institute at Geisinger in Lewisburg, Pennsylvania. She spoke with Spectrum about those barriers and how clinicians and geneticists can work together to 
overcome them.

\section{Spectrum: Why is genetic testing for autism important?}

Brenda Finucane: The purpose of diagnostic genetic testing for children and adults with autism is to try to identify an underlying genetic cause of autism. Knowing a specific genetic cause can sometimes help guide treatment. For example, a result might reveal that a person should be monitored for certain medical conditions, such as kidney problems, known to be associated with a genetic disorder. Genetic testing can also provide information about inherited causes of autism and the chance that future children in the family will also have the condition.

For some people, knowing that autism resulted from a genetic medical condition can reduce feelings of guilt or stigma; the genetic condition isn't something anyone caused. And once they have a diagnosis, many are very grateful, because it plugs them into a group of other people who share the same specific genetic disorder.

\section{S: Who is undergoing genetic testing now?}

BF: Recommendations for genetic evaluations of people with autism have been in place for many years, but surveys show that the majority never undergo comprehensive testing. Those who do are often young children seen at major university hospitals, and typically, testing is done through referral to a medical genetics department. The majority of people living with autism remain untested and unaware of the cause of their autism.

Because genetic testing is clinically recommended for people with autism, it should be offered as part of their medical care, and it is often covered by health insurance. In addition, some research projects, such as SPARK, do genetic testing and are returning results to families when they find a diagnosis. [SPARK is funded by the Simons Foundation, Spectrum's parent organization.]

\section{S: What is keeping most people with autism from getting diagnostic genetic testing?}

BF: Sometimes specialists assume that a child or adult with autism has already been referred by other healthcare providers for genetic testing, and they don't take it on themselves to do it.

It's not uncommon to encounter older children and teenagers with autism who have been to many different specialists - neurologists, psychiatrists, developmental pediatricians - and the value of genetic testing has never been explained to them.

A 2017 study found that many child neurologists and developmental pediatricians did not order recommended genetic tests for children with autism, or they ordered inappropriate or outdated tests that did not reflect current standards. More recently, a 2020 study found that only 3 percent of autistic children had completed recommended genetic testing, citing obstacles such as their 


\section{Spectrum | Autism Research News}

https://www.spectrumnews.org

clinician's lack of familiarity with ordering genetic tests.

\section{S: What are your recommendations for increasing access to diagnostic genetic testing?}

BF: We need to make sure that specialists - particularly neurologists, psychiatrists and developmental pediatricians, who see the majority of people with autism - are all aware of the current guidelines for genetic diagnostic testing. And we have to make very practical strategies for how they can seamlessly do genetic testing during a busy day.

Ideally, we'd love to see these specialties come together and collaboratively come up with a new set of guidelines. In the interim, people should look to what is recommended by the genetics experts, the American College of Medical Genetics and Genomics.

\section{S: What can clinicians do to try to meet those guidelines?}

BF: Clinicians need to establish a protocol for getting everyone appropriately tested. They should choose one lab and send all the samples there. Talk with the lab about streamlining the process and having a plan for insurance.

Clinicians should reach out to a genetics office or a medical genetics department in their institution or their region to set up a referral pipeline. They can ask a geneticist to work with the office staff and help set up an appropriate, vetted letter to send to any patients who have negative results. And then only refer the positive tests and the tests that identify variants of uncertain significance to the medical genetics department.

\section{S: What kind of genetics training do you think clinicians need to have?}

BF: I think that historically, we've gone wrong by trying to turn these specialists into 'mini geneticists.' Instead, we should train them on the practical things: Here's how you set up your insurance, here's how you set up your pipeline to send out samples to labs and refer the patients who need further testing.

They should know why this is important. Give them actual cases — for example, a person with tuberous sclerosis who has autism, who is now actually on a medication that is reducing the kidney tumors they get, which is one of the biggest causes of death in that syndrome. It's about why this is important for families, why it's important from a clinical standpoint, and how it's going to help drive therapeutics very specifically in the future.

\section{Cite this article: https://doi.org/10.53053/JBHQ5067}

